\title{
ELECTRONIC STRUCTURE OF COBALT-NICKEL MIXED OXIDES
}

\author{
Stephan Schmidt*, Dieter Schmeißer \\ Brandenburg University of Technology Cottbus, Konrad-Wachsmann-Allee 1, D-03046 Cottbus. \\ schmiste@tu-cottbus.de
}

Keywords: transition metals, oxides, resonant photoemission, NEXAFS

\section{Introduction}

Replacing cobalt by large amounts of nickel is considered to reduce costs in Lithium-Ion-Batteries while maintaining their performance. We investigate the electronic structure of cobalt-nickel compounds because of its direct impact on battery operation.

\section{Experimental details}

Thin cobalt-nickel oxide films on metal substrates were prepared by dropping/spin-/spray-coating from aqueous nitrate solutions followed by subsequent annealing in air and UHV. The following cobalt-to-nickel ratios have been investigated: 50/50, 40/60, 30/70, and 20/80. The results were compared with the pure oxides and magnetron sputtered $\mathrm{LiCoO}_{2}$ reference samples ${ }^{[1]}$. Spectroscopic measurements on these samples were carried out at the U49/2 beamline at BESSY II, Berlin ${ }^{[2]}$. All samples were characterized by means of photoelectron and $\mathrm{x}$-ray absorption spectroscopy (PES, NEXAFS). XPS of the transition metal $2 p$ regions was performed to calculate the cobalt-to-nickel ratio and to reveal the existence of chargetransfer ground states and different oxidation states. Resonant photoemission probes the density of occupied states and was used to distinguish the contributions of the transition metal $3 \mathrm{~d}$ and the oxygen $2 \mathrm{p}$ states in the valence region. NEXAFS at the oxygen $\mathrm{K}$ and transition metal $\mathrm{L}_{2,3}$ edges respectively, projects the density of unoccupied states just above Fermi energy. NEXAFS spectra were taken in the total electron yield mode (TEY).

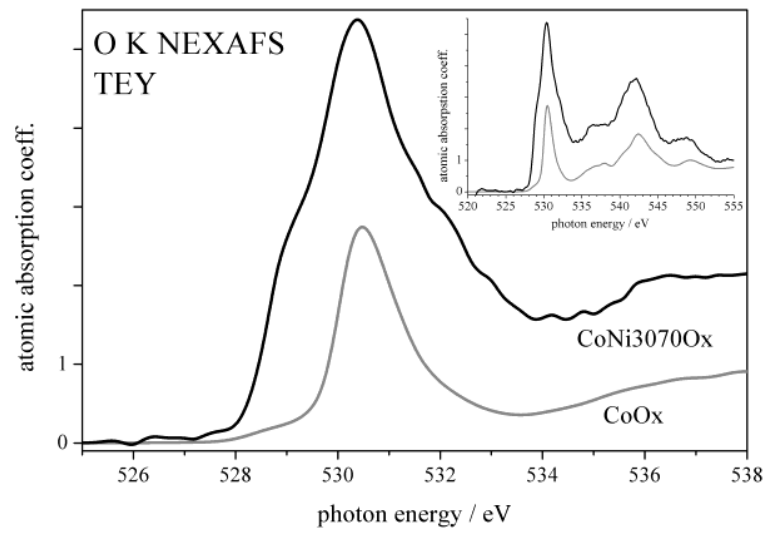

Fig. 1 Oxygen K edge NEXAFS of a mixed oxide with relative amounts of 30\% cobalt and $70 \%$ nickel (bold) and the pure cobalt oxide

\section{Results}

The admixture of nickel was expected to increase the content of bivalent cobalt ions with respect to the pure cobalt oxide which is of mixed valence including $\mathrm{Co}^{2+}$ and $\mathrm{Co}^{3+}$. Though the differences between the two species in the XPS signals are limited to the width of the main peaks and broad satellites one can easily follow the transition using NEXAFS at the cobalt $\mathrm{L}_{3}$ edge. This is possible because of its rich multiplet structure that can be reproduced in good agreement with the experiment by the semi-empirical software CTM4XAS ${ }^{[3]}$ using parameters from cluster calculations. The comparison of the $\mathrm{O} K$ NEXAFS signals indicates features below the main edge that have different intensities with respect to the cobalt-tonickel ratio. For the mixture with the strongest intensity of this feature a complete set of energy dispersive curves (EDC) was acquired. In a contour plot of these curves we can distinguish between features at constant binding or constant kinetic energy. This gives us the opportunity to study the phenomena connected with resonant excitation. The behaviour of the pre-edge region as well as the evolution of the strongest multiplet components of the $\mathrm{O}$ KLL auger lines with excitation energy could be analysed.

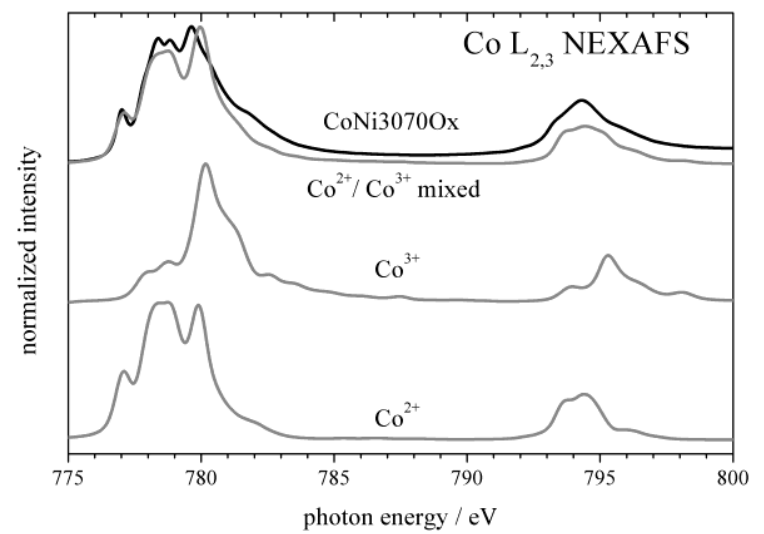

Fig. 2 Cobalt $\mathrm{L}_{2,3}$ edge NEXAFS CTM calculations of $\mathrm{Co}^{2+}$ and $\mathrm{Co}^{3+}$ in comparison with the experimental spectrum of the sample CoNi3070Ox (bold)

\section{References}

[1] D. Schmeißer, S. Schmidt, G. Seibold, G.

Cherkashinin, W. Jaegermann, ECS Trans., 25 (2010) 37-45.

[2] D. Schmeißer, P. Hoffmann and G. Beuckert, Materials for Information Technology, ISBN 1-85233-941-1, (2005) 449-460.

[3] E. Stavitski, F. M. F. de Groot, Micron, 41 (2010) 687. 